Chemical Physics 128 (1988) 209-217

North-Holland, Amsterdam

\title{
EXCITON REACTIONS IN ULTRATHIN MOLECULAR WIRES, FILAMENTS AND PORES: A CASE STUDY OF KINETICS AND SELF-ORDERING IN LOW DIMENSIONS
}

\author{
Raoul KOPELMAN, Stephen J. PARUS and Jagdish PRASAD \\ Department of Chemistry, University of Michigan, Ann Arbor, MI 48109, USA
}

Received 18 February 1988; in final form 2 April 1988

\begin{abstract}
When are molecular wires thin enough to show one-dimensional exciton kinetics? Cylindrical naphthalene wires (5-5000 nm radius) show a definite one- to three-dimensional transition (about $25 \mathrm{~nm}$ for triplet excitons at $4 \mathrm{~K} ; 40 \mathrm{~nm}$ at $77 \mathrm{~K}$ ). Nuclear channel pore membranes (polycarbonate) serve as templates and calibrators. The triplet exciton migration (multiple hopping) length is $50-100$ molecules. The closest neighbor distribution is used as an order criterion. Steady-state simulations of the reactions: $\mathbf{A}+\mathbf{A} \rightarrow \mathbf{0}$ and $\mathbf{A}+\mathbf{A} \rightarrow \mathbf{A}$ in low-dimensional media give non-random reactant distributions, c.g. Wigner-like rather than Poissonian spacings in one dimension. Effectively, a dynamic, quasi-ordered superlattice of excitations is created (excitation grating with a $5 \mathrm{~nm}$ spacing). Experimental verification involves a new technique: excitation time modulation. Porous glass (Vycor) is shown to have an effective one-dimensional channel topology. Naphthalene powder and isotopic alloys also show nonrandom steady-state exciton distributions.
\end{abstract}

\section{Introduction}

Excitation kinetics allows us to study paradigms of transport in disordered films, polymers and membranes as well as paradigms of heterogeneous chemical kinetics. It is also a tool for studying the topology, morphology and structure of molecular aggregates, strands, pores and domain boundaries. Newly developed techniques of exciton luminescence are based on the anomalous energy diffusion and anomalous excitation population distributions in confined and/ or low-dimensional media. The ideal testing grounds for such techniques are one-dimensional crystals. We present below close experimental approximations of such systems. We also present simulations on both ideal one-dimensional lattices and approximations thereof representing the experimental systems. In particular we show that excitations, and reactants in general, are not distributed at random, but achieve a stable, self-ordered distribution. For example, for the $A+A \rightarrow A$ reaction in one dimension, the random Poissonian nearest-neighbor distribution is replaced by a skewed-Gaussian distribution. This gives rise to a mesoscopic scale, with a structure resembling a transient grating.

Solid-state and stochastic problems in one dimen- sion have been of long-standing theoretical interest [1-5]. Currently, electronics in thin wires are of much theoretical and practical interest [6-9]. The theoretical enigmas (localization, mesoscopic phenomena, boundary effects) are compounded by experimental difficulties: minute currents, heat dissipation, shorts, non-uniformity and suspect testing procedures. Many of these difficulties are not present for Frenkel excitons. There are no Coulomb repulsions and for triplet excitons the interactions are extremely short-ranged and the surface effects are minimal $[4,10]$. Moreover, triplet excitons are already localized in the bulk [11] and thus there is no localization-delocalization transition of cross-over. Experimentally one can rely on optical measurements which are as simple for thin wires as for the bulk. Furthermore, sample uniformity or continuity is not a crucial factor. One can thus concentrate on the mesoscopic properties of interest, stemming from the confinement of the excitons inside a thin "wire".

Recently, porous materials and "fractal" networks have also been of much interest [12-15]. The difference between a fractal network and a quasi-one-dimensional network is not often all that clear [12-16]. Energy transfer [13,14] and exciton kinetics [15] have been used for the characterization of such net- 
works (e.g. pore networks of porous media). Understanding the characteristics of truly one-dimensional networks and the effects of sample diameter is thus of practical interest. Furthermore, molecular or polymeric chains, fibers, filaments and networks exist in most synthetic, natural and biological organic systems, from organic conductors to neuron transmitters. Molecular exciton kinetics in thin filaments are of relevance to all these systems.

We note that our systems differ significantly from previously studied quasi-one-dimensional systems [17-20]. The latter are essentially two- or threedimensional systems with highly anisotropic excitonexchange interactions. Thus, for a short time the exciton is confined in one dimension. However, there is always a finite probability of moving along other directions (interchain hopping), resulting in a twoor three-dimensional behavior over longer times (this usually confines the measurements to ultrashort times). Moreover, the phonons and exciton-phonon interactions in these systems are seldom one-dimensional. In contrast, our systems are truly one-dimensional over long times and there is no escape or tunneling out of the thin, one-dimensional systems. (Our ultrathin wires are obviously three-dimensional on extremely short time scales.)

It has been demonstrated [21] that the reaction $A+B \rightarrow 0$, in low dimensions, has a segregated, partially ordered steady state. It is demonstrated here that the simpler $\mathrm{A}+\mathrm{A} \rightarrow$ product reactions also lead to ordering, i.e. non-random closest neighbor distributions. This ordering accounts for the non-classical steady-state reaction kinetics demonstrated earlier [22-26] for low-dimensional $(d \leqslant 2)$ media. It also makes possible a simple, but powerful, new experimental technique for the characterization of low-dimensional or disordered media $[27,28]$. This ordering process also accounts for the anomalous kinetics of unstirred batch ("big-bang") reactions in low dimensions [29-37].

The rate-law for the elementary $A+A \rightarrow 0$ or $A+A \rightarrow A$ steady-state reaction is given by

$$
R=K \rho^{x}
$$

where $R$ is the steady-state creation rate, $\rho$ the density, $K$ a rate constant and $X$ the reaction order. Classically, $X=2$. For the one-dimensional lattice it was found [22,23] that $X=3$, and, in general for fractal domains, $X=1+d_{\mathrm{s}} / 2\left(d_{\mathrm{s}}<2\right)$, where $d_{\mathrm{s}}$ is the spectral dimension $[23,26,38,39]$. The interpretation of these high $X$ values is not straightforward, even though they have been linked to the time-dependent rate coefficients for transient reactions $[23,26]$. For monodisperse islands and percolation clusters, extremely high $X$ values were found, both experimentally [35] and by simulation [25]. On the other hand, replacing the strictly random creation of $A$ particles with a geminate random creation reduces $X$ back to two in one dimension [22,37] and down to one for islands [37]. For a uniformly random (in one dimension) distribution of particles, the probability $p$ of finding a nearest-neighbor pair (for $\rho \rightarrow 0$ ) is $p \sim \rho^{2}$. Hence the instantaneous reaction rate is $R \sim p \sim \rho^{2}$. An order $X \neq 2$ implies the absence of a random (Poissonian) particle distribution, i.e. the presence of partial ordering. For instance, an evenly spaced population or a population with a skewed-Gaussian nearest-neighbor kinetic distribution given by $K(r)=\alpha r \exp \left(-\beta r^{2}\right)$ results in $R \sim \rho^{3}$, compared with $R \sim \rho^{2}$ derived from the Poissonian (Hertzian) distribution $H(r) \sim \exp (-\gamma r)$. The existence, nature and consequences of such anomalous distributions are addressed below.

Most of our previous studies [15,26,34-37] employed a "long-time technique", i.e. a study of longtime phosphorescence and delayed fluorescence decays. In section 2 we employ this technique on our most ideal one-dimensional naphthalene samples. In section 3 we test out a novel "short-time technique", which is explicitly based on the phenomenon of kinetic self-ordering. Its utility and theoretical implications are first demonstrated via computer simulations. Subsequently, experiments are presented on one-dimensional as well as other low-dimensional or disordered systems. In section 4 we compare and summarize the findings from both techniques.

\section{Long-time excitation kinetics in molecular wires and filaments}

Exciton transport is usually monitored via the kinetics of trapping or annihilation $[11,15,18,26]$. The kinetic process may be unary, pseudo-unary or bi- 
nary (monomolecular, pseudo-monomolecular or bimolecular in chemical language), e.g., trapping, heterofusion or homofusion, respectively $[4,15,26]$. In all these cases the "rate constant" (instantaneous reactive collision probability per unit density) is given by [26]

$k \sim \mathrm{d} S / \mathrm{d} t$,

where $S$ is the mean number of distinct lattice sites visited by a single exciton (in the absence of reactive processes). We note that eq. (2) is valid for all topologies, in contrast to the expression $k \sim D$ ( $D=$ diffusion constant) which is valid only for threedimensional (homogeneous) lattices. To a very good approximation one has [26]:

$S \sim t^{f}, \quad \frac{1}{2}<f<1 ;$

$k \sim t^{-h}, \quad \frac{1}{2}<h=1-f<1$.

At long times $h=0$ for three-dimensional lattices and $h=\frac{1}{2}$ for one-dimensional lattices. We note that for fractal lattices $h=1-d_{\mathrm{s}} / 2$ where $d_{\mathrm{s}}$ is the spectral dimension [26,37-39]. The pragmatic questions we pose are: How thin has a wire to be to give one-dimensional behavior $\left(h=\frac{1}{2}\right)$ ? What is the nature of the crossover from three-dimensional to one-dimensional behavior? What does this crossover depend on? What can we learn from it? What is the use of thin exciton wires?

We note here that in perfect crystalline samples the excitons move freely (at random, due to phonons), resulting in binary (bimolecular) exciton-exciton annihilation [26,36,37]:

$\mathrm{A}+\mathrm{A} \rightarrow h \nu$,

where $h \nu$ designates fluorescence ("delayed"). Thus the annihilation rate $R$ and the fluorescence rate $F$ are given by [36]

$F \sim R=k \rho_{A}^{2}$,

where $\rho_{\mathrm{A}}$ is the free exciton density. However, in most real samples [15], a fraction of the free excitons (A) are quickly trapped, giving a roughly constant density $\rho_{\mathbf{A}^{\prime}}$ of trapped excitons ( $\left.\mathrm{A}^{\prime}\right)$, resulting in a pseudounary (pseudo-monomolecular) annihilation reaction and rate $[15,40]$ :

$\mathbf{A}+\mathbf{A}^{\prime} \rightarrow h \nu$,
$F \sim R=k^{\prime} \rho_{\mathrm{A}}, \quad k^{\prime}=k \rho_{\mathrm{A}}$.

In addition, the triplet excitons undergo natural decay (lifetime $\tau$ ):

$\mathrm{A} \rightarrow h \nu ; P=\tau^{-1} \rho_{\mathrm{A}}$.

The overall results are thus:

$k \sim F / P^{n}, \quad n=1,2$,

where $n=2$ is for perfect (trapless) samples and $n=1$ is for real samples (with traps). We note that for three-dimensional samples $k$ (and $\log k$ ) is expected to be constant in time while for one-dimensional samples $k \sim t^{-1 / 2}$ and $\log k \sim \log t$ (with a slope of $-1 / 2)$.

An important fine point concerns the validity of relations such as $k \sim t^{-1 / 2}$ or eqs. (1) and (4) in the short and intermediate time domain. The standard theoretical derivations $[29,30,36]$ emphasize that these are asymptotic relations $(t \rightarrow \infty)$. However, explicitly or implicitly, at $t=0$ the ensemble of reactants has a random (Poisson) distribution and a classical reaction rate. By contrast, in our experiments $[15,36,40]$ the exciton ensemble is prepared under steady-state exitation where the source is turned off at $t=0$. We show below (section 3 ) that the resulting distribution is a stable, self-ordered distribution. For such a distribution the rate law $R \sim \rho^{3}$ and its analogs are valid for all $t>0$ and not only for $t \rightarrow \infty$.

The optical arrangement and sample preparation have been described before $[15,40]$. The only significant change involves the use of channel-pore ("Nuclepore") \#1 polycarbonate membranes. These $6 \mu \mathrm{m}$ thick membranes come with well isolated, cylindrical pores (fig. 1). While a given membrane has uniform pore diameters, membranes with different pore diameters are available and we used them in the range of $10 \mathrm{~nm}(100 \AA)$ to $1 \mu \mathrm{m}(10000 \AA)$. Some typical results are shown in fig. $2(T=4 \mathrm{~K})$ and fig. $3(T=77$ K). We note that only the pseudo-unary model $(n=1)$ resulted in linear slopes. The binary model $(n=2)$ cannot be fitted with straight lines and, moreover, results in non-constant $k$ curves even for the thickest wires $(1.2 \mu \mathrm{m})$. The totality of the $h$ values (negative slopes), for all wires (each at 4 and 77 $\mathrm{K})$, is given in fig.4.

\#1 Nuclepore Corp., Pleasanton, CA; for similar applications to wire fabrication, see ref. [41]. 


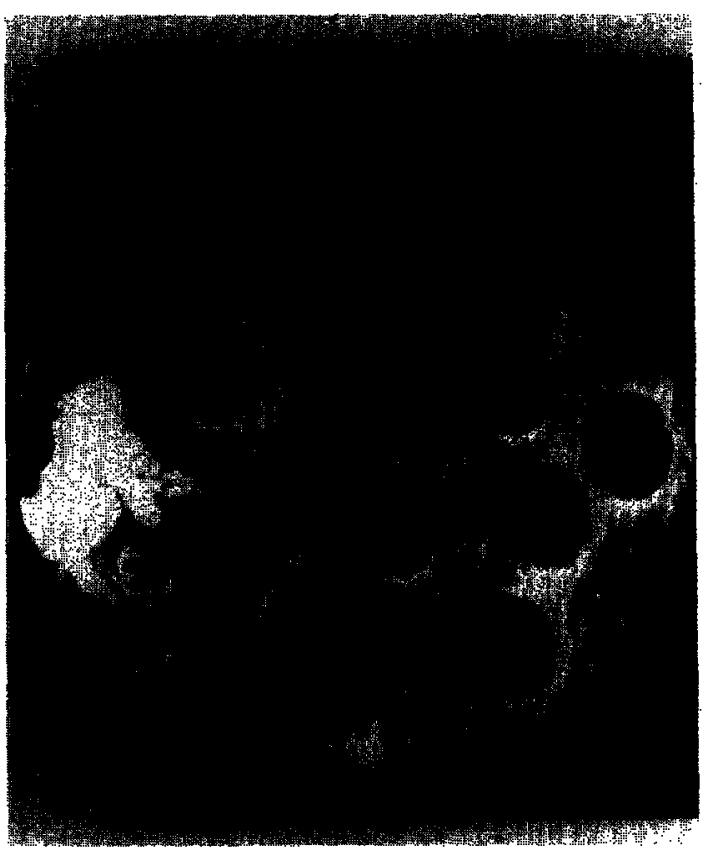

Fig. 1. Channel-pore membrane: polycarbonate ("Nuclepore"). Enlargement about $\times 10^{4}$. Pore length $6 \mu \mathrm{m}$.

We observe that the thinnest wires yield a value $h \approx 0.5$, while the thickest wires give $h \approx 0$, for both temperatures. Actually, extrapolating to zero diameter, $h \rightarrow 0.49 \pm 0.02$. On the other hand, for micron-

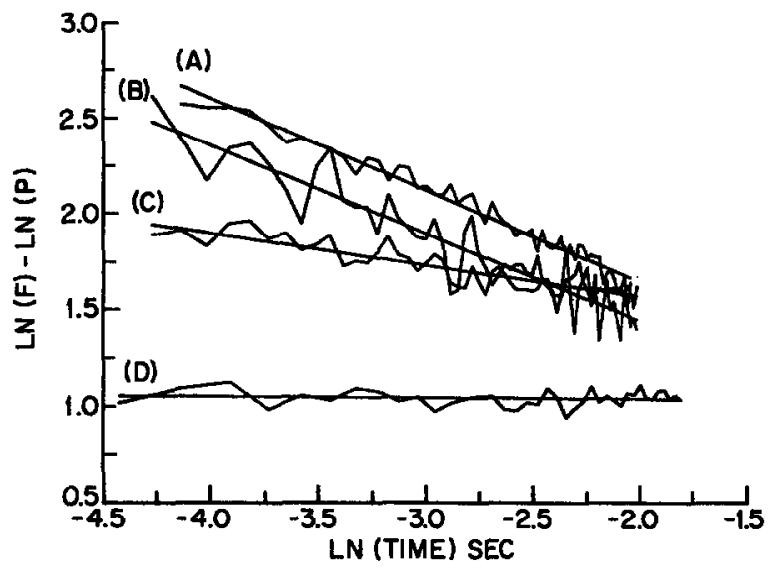

Fig. 2. Annihilation rate coefficient $R=F / P$ versus time on a lnIn scale, for naphthalene filled channel-pore polycarbonate membranes at $T=4 \mathrm{~K}$. The pore radii are (A) $75 \AA$, (B) $150 \AA$, (C) $250 \AA$ and (D) $400 \AA$. Note that the trapped (bound) exciton phosphorescence is excluded via an interference filter (centered at the free exciton peak).

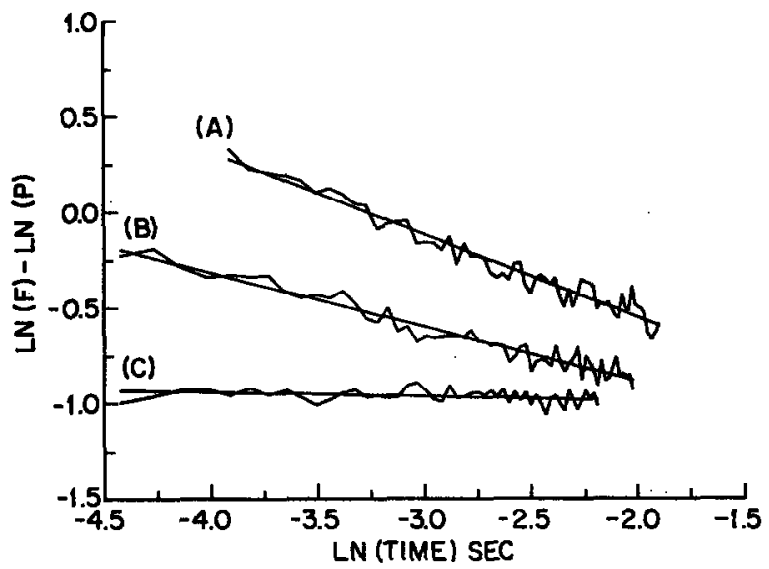

Fig. 3. Annihilation rate coefficient $R=F / P$ versus time on a lnIn scale, for naphthalene filled channel-pore polycarbonate membranes at $T=77 \mathrm{~K}$. The pore radii are (A) $250 \AA$. (B) $400 \AA$ and (C) $1000 \AA$.

sized wires $h \rightarrow 0.02 \pm 0.02$. These two limiting values are in excellent agreement with the theoretically expected values of $h=1 / 2$ and $h=0$, respectively $\#$. The crossover (from $h \approx 0$ to $h \approx \frac{1}{2}$ ) occurs at diameters of about 500 to $800 \AA$ at 4 and $77 \mathrm{~K}$, respectively. The crossovers are relatively sharp and their temperature dependence is relatively mild. The higher value at higher temperatures is consistent with a somewhat

\#2 We note that the finite width of the thin wire does not invalidate the $h=1 / 2$ value. Simulations on a "bundle" of three interconnected lines ("Toblerone" shaped) give $h=0.5$ [42].

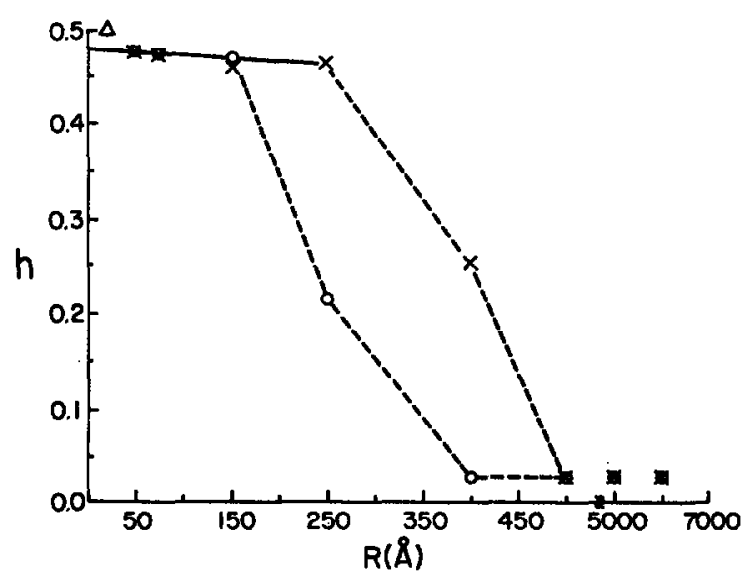

Fig. 4. Exponent $h$ versus wire radius, $r$ (in ångström), at $4 \mathrm{~K}$ (O) and $77 \mathrm{~K}(x)$. The $20 \AA$ point $(\Delta)$ is for porous Vycor [15]. Note break in scale. 
faster hopping rate. In our interpretation the crossover radius is roughly consistent with the average cruising range $\lambda$ (endpoint to endpoint distance) of the exciton, within its lifetime.

An indirect, rough estimate for the naphthalene cruising, range in similarly prepared samples was given [15] as $\lambda=1000 \AA$. This is in excellent agreement with our present result $(\lambda \approx 500 \AA)$. Obviously, for wires with radius $r \gg \lambda$ the excitons do not "feel" the pore boundaries while for $r \ll \lambda$ the excitons are severely confined along two of the three directions. We note that the polycarbonate excitation energy values are so much higher than those of naphthalene that there is a vanishing probability for barrier crossing or tunneling $(\Delta E>100 k T$ even at $77 \mathrm{~K})$.

Regarding previously studied random pore membranes ("Gelman") [15], we notice that these are prepared by an entirely different process, resulting in an apparent self-similar distribution of pore sizes (see picture in ref. [26]). As pointed out before [15], the exciton annihilation method is heavily biased towards small pores. Thus the previously derived $h$ values can now be calibrated in terms of weighted pore sizes (for the nylon, acetate, etc. pores of ref. [15]). Specifically, a range of $h$ values between 0.1 and 0.3 can be interpreted in terms of a weighted pore size distribution of a few hundred ångström. This is in contrast to the much larger nominal pore sizes, based on filtration experiments. On the other hand, it is possible to retain a fractal-like interpretation [15], where $h=1-d_{\mathrm{s}} / 2$ and $d_{\mathrm{s}}$ is an effective spectral dimension. This also explains how different $h$ values may be obtained by different experimental methods. For instance, the photodimerization method [40] which is based on the diffusion of excited molecules in solution, does result in a somewhat higher range of $h$ values for the same random porous membranes (probably due to a different weighting). An interesting exception to the random pore membranes appear to be the natural (cellulose) filter papers. These are not likely to contain a self-similar distribution of minipores. The $h=0$ result [15] points towards nearly cylindrical pores, with a diameter distribution that has a cut-off higher than $1000 \AA$.

Of particular interest is the resolution of the porous glass (Vycor) dilemma [15]. The nature of the pore network has been highly controversial [12-16]. It has been argued on the one hand that it is a random (percolation-like) network with a fractal dimension on the order of two [13]. On the other hand it has also been argued to be non-fractal but essentially onedimensional $[12,15,16]$. Based on the exciton kinetics technique it was argued [15] that the effective spectral dimension is 1.05 , i.e. effectively one-dimensional. Our present study uses the same approach for "calibrated" cylindrical pores which are obviously non fractal and one-dimensional. We have included the older [15] Vycor glass measurements as a data point in fig. 4. It essentially falls on the same curve as the new, polycarbonate data. The Vycor data are thus totally consistent with a one-dimensional pore topology $[42,43]$.

In summary: (1) We have produced cylindrical molecular crystal wires down to a radius of $5 \mathrm{~nm}$. (2) The recombination process involves free and bound excitons ("heterofusion"). (3) The triplet exciton kinetics fits a multiple-hopping model. (4) The overall migration range is about $25 \mathrm{~nm}$ at $4 \mathrm{~K}$ and $40 \mathrm{~nm}$ at $77 \mathrm{~K}$. (5) The long-time exciton transport is strictly one-dimensional in the ultrathin wires. (6) The fractal-like kinetics model works well in a low-dimensional non-fractal system. (7) The porous-glass (Vycor) channels are described well by a non-fractal, quasi-one-dimensional topology. (8) The exciton annihilation method appears to be a reliable tool for probing spectral dimensions and low-dimensional topologies.

\section{Short-time excitation kinetics in one-dimensional and fractal-like samples: self-ordering}

In this section we demonstrate explicitly the phenomena of kinetic ordering in low dimensions for the simplest binary reaction $A+A \rightarrow 0$ or $A+A \rightarrow A$. $A$ new, mesoscopic, length-scale (the most probable closest neighbor distance) emerges. This not only explains the anomalous long-time reaction kinetics described above but also gives rise to a new short-time technique which, first of all, demonstates the excitation self-organization, and secondly, provides a new

\footnotetext{
\#3 We believe that the very recent report [43] of a spectral dimension of 1.1 is also consistent with a quasi-one-dimensional pore topology.
} 
method for the characterization of low-dimensional structures.

\subsection{Simulations}

The experimental exciton reaction: triplet + triplet $\rightarrow$ fluorescence was simulated by $A+A \rightarrow 0$ and $A+A \rightarrow A$ random walks on a variety of lattice surfaces. Comparisons were made between different initial conditions. Steady-state conditions were generated by adding several new walkers per step until the total number of walkers was constant. Pulsed conditions were then studied by adding in one step the same number of walkers that exist at steady state to produce an initial uniformly random distribution. In both cases, after stopping the addition of walkers $(t=0)$, the number of walkers or reactants remaining at time $t$ (equivalent experimentally to phosphorescence) and the number of annihilations or reaction rate at time $t$ (equivalent experimentally to delayed fluorescence) were monitored. While the initial $(t=0)$ global densities are equal for both forms of reactant generation, this does not guarantee equal reaction rates at $t \geqslant 0$ or equal global densities at $t>0$. Examples are given in fig. 5 which is discussed further below.

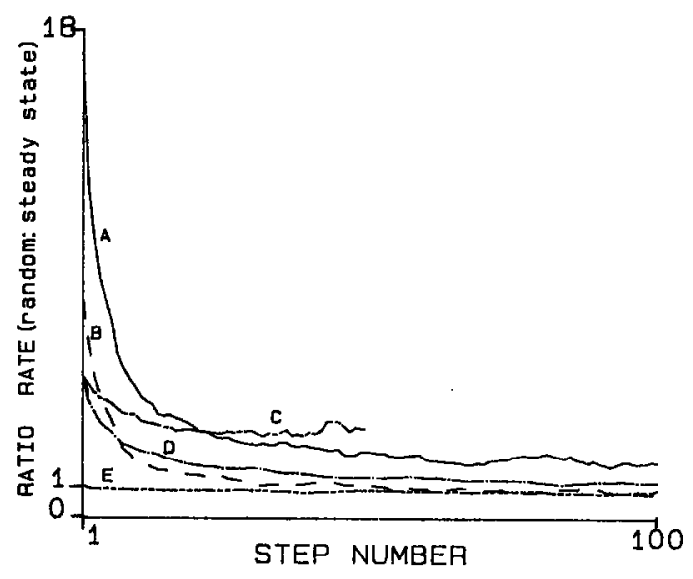

Fig. 5. Ratio of annihilation reaction rates $R_{\mathrm{ur}} / R_{\mathrm{ss}}$ (rate for uniformly random pulse over rate for steady-state generation) versus step number for the simulation $A+A=0$ on various lattices. Total number of sites $=30000$. (A) One-dimensional islands (20 sites each). (B) One-dimensional continuous chain. (C) Three-dimensional islands $(3 \times 3 \times 3$ sites each $)$. (D) Three-dimensional percolation clusters (cubic; $40 \%$ occupation, all clusters). (E) Three-dimensional cube.
The spatial and temporal aspects of a distribution of reacting particles (excitons) are related. The simulations show this most clearly for a one-dimensional lattice. Fig. 6 gives the distribution $D(l)$ of interparticle (closest neighbor) gaps in terms of gap-lengths (l). For a Poissonian (uniformly random) distribution of particles the most probable gap is the shortest one ( $\bar{l}=1$ lattice unit), giving a quasi-exponential $D(l) \sim \exp (-\beta l)$ one-dimensional [44] distribution of gaps. This is no longer true for the steady-state distribution. Here there is a most probable gap-length $(T>1)$ with a skewed exponential $(D) l \sim l \exp (-\beta l)$ distribution. Similar $A+A \rightarrow A$ simulations give a skewed Gaussian (see above), Wigner-like distribution $[45,46]$. We note that experimentally, the random distribution represents a pulse-created exciton distribution. Allowing each one of the two realizations (pulsed and steady state) to relax reactively (with no further supply of particles), one finds two different reactive decay curves in spite of the equality of populations for both realizations. The initial decay rate will differ most drastically as it is only determined by pairs with a gap $l=1$. There are many more such $l=1$ gaps for the pulsed distribution than for the steady-state distribution, the ratio being about ten for the one-dimensional lattice (see fig. 6). Fig. 5, curve

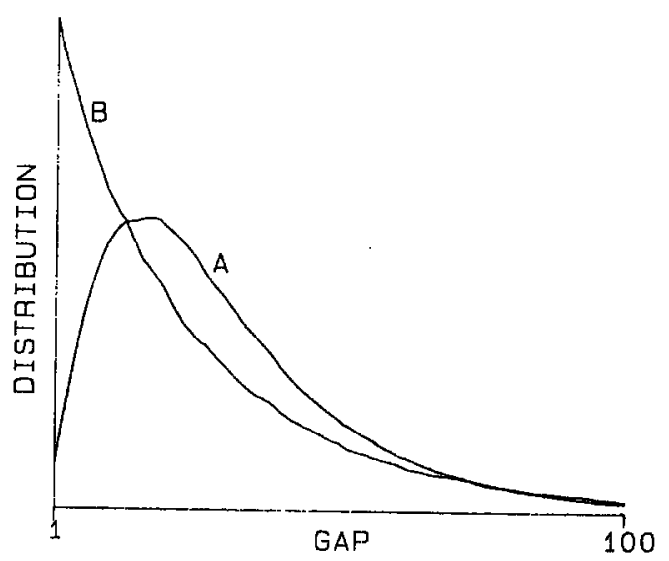

Fig. 6. Distribution $D(l)$ of the interparticle closest neighbor distance (gap) $l$ in lattice units for each particle on a one-dimensional line 30000 long: $(A)$ Steady state $(A+A \rightarrow 0)$ after 2000 steps of adding two particles per step (density $=2 \%$ ), averaged over 1000 realizations. (B) Uniformly random distribution of the same number of particles as in (A), with no reaction steps taken. Note that for gap length $l=1$ the gap population is 10.7 that of case $(A)$. 
(B) plots the ratio of reaction (fusion) probability as a function of time for the one-dimensional lattice. At $t=0$ this ratio is about eight. The two ratios are related (but not equal). In principle, curve (B) of fig. 5 carries the information given in fig. 6 . It is obvious from fig. 5 that the most significant steady-state ordering occurs for one-dimensional-islands (filaments). On the other hand, there is no ordering effect for a cubic lattice. Other topologies show an intermediate behavior (which is highly specific). The simulations of fig. 5 give the temporal aspect, which is closest to our experiments. We emphasize here that, for each topology, the initial populations (steady state and pulsed) are strictly equal. This is also the key for the design of meaningful experiments (see below).

The anomalous values of the reaction order $X$ (eq. (1)) can now be addressed. With $X=3$ (the one-dimensional result), there is an extra power in the $\rho$ dependence. This can be related to the extra lengthscale $(l)$ that enters the problem. Obviously, $l \sim \rho^{-1}$. It appears that now $R \sim p \sim \rho^{2} l^{-1} \sim \rho^{3}$. We interpret this as meaning that the probability of having a pair at distance $l$ is $\rho^{2}$, but at a distance of unity this probability is scaled down ${ }^{\# 4}$ by $l$.

Analogous arguments can be made for other lowdimensional media $(d \leqslant 2)$. The interesting question remains: What determines the critical dimensionality for this self-ordering effect? Is it the same as for the segregation in the A+B case [21]? Obviously, above this critical dimension the diffusional self-stirring [37] efficiently re-randomizes the reacting particle distribution, not only for steady-state reactions, but also for transient ("pulse", "batch", "big-bang") reactions.

\subsection{Experiments}

Triplet fusion experiments were performed on naphthalene impregnated porous glass (Vycor), naphthalene powder and perfectly crystalline isotopic alloys of naphthalene $\left(\mathrm{C}_{10} \mathrm{H}_{8}: \mathrm{C}_{10} \mathrm{D}_{8}\right)$. Purification, preparation and other experimental procedures were the same as in previous studies [15]. The time dependence of the naphthalene triplet-trip-

\footnotetext{
\#4 Rigorously, a skewed Gaussian or skewed exponential distribution in one-dimension gives a $\rho^{3}$ global rate law, based on a local $\rho \omega$ law, where $\omega$ is the gap $(g)$ distribution for $g \rightarrow 0$.
}

let exciton annihilation reaction rate was monitored by delayed fluorescence emission. Phosphorescence emission corresponds to the instantaneous triplet reactant concentration (global density). The dependence of the relative decay rates and intensities of the emissions on initial excitation duration was examined. A random population of excitons was produced by pulsed excitation ( 5 ms duration) from a mechanically shuttered xenon arc lamp. The steady-state population was created by leaving the shutter open for several seconds (which is longer than the time required to establish a constant phosphorescence signal). Neutral density filters were used to give a phosphorescence intensity at time zero (the closing time of the shutter) equal to that of the pulsed mode. This ensures equal initial global exciton densities. However, it is not sufficient to assure equal annihilation kinetics. Actually, both the phosphorescence and the delayed fluorescence decays are quite different for the pulsed and steady state cases. Fig. 7 shows the results for naphthalene embedded porous glass. Similar results are obtained for naphthalene powder and for low concentration naphthalene single crystal isotopic alloys $\left(\mathrm{C}_{10} \mathrm{H}_{8}\right.$ below percolation [26,34-

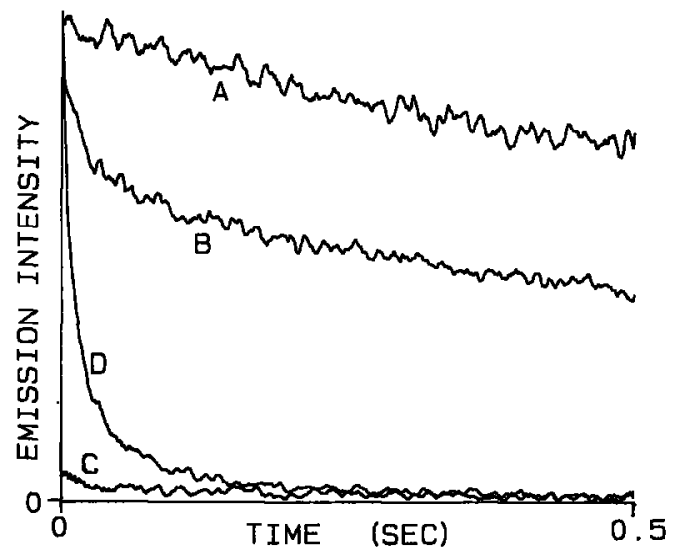

Fig. 7. Relative intensities of phosphorescence and of delayed fluorescence decays from naphthalene in porous Vycor glass at $18 \mathrm{~K}$ following steady-state (11 s duration) or pulsed $(20 \mathrm{~ms}$ duration) excitation ( $3.19 \mathrm{~nm}$ ). (A) Phosphorescence from steady-state excitation with a number 2.0 neutral density filter to equalize its initial intensity to that of (B). (B) Phosphorescence from pulsed excitation. (C) Delayed fluorescence from conditions in (A). (D) Delayed fluorescence from conditions in (B). Note that the intensity scale for (C) and (D) is different than for (A) and (B). 
36]). On the other hand, no such differences in intensities or decay rates are observed for high concentration alloys (above percolation) or for nearly perfect naphthalene crystals. Above percolation, the single crystal alloy exhibits classical rather than geometrically restricted transport characteristics. This range of behaviors is shown in fig. 8 .

From figs. 5 and 8 it appears apparent that the porous $\mathrm{Vycor}$ naphthalene filaments have an effectively one-dimensional, wormlike topology. Similar conclusions were obtained from long-time decays of phosphorescence and delayed fluorescence $[15,47]$. The long-time technique is based on the anomalous diffusion (compact random walk) in low dimensions while the current short-time technique is based on the anomalous distributions of the reactive populations at low dimensions. Obviously, there is a direct relation between the compactness of the diffusion and the ordering of the reactants. However, the longtime decay experiments are weighted more heavily by reactions on longer naphthalene filaments (worms) while the current short-time decays may be weighted more heavily by reactions on shorter filaments. We estimate the filament lengths at 200-1000 $\AA$ or longer, with an effective one-dimensional topology. The Vycor pore topology is thus quasi-one-dimensional over a range of $200 \AA$ and possibly $1000 \AA$ or longer. This is consistent with some literature conclusions [12] but not with others [13]. It is evident from figs. 8 and 5 that a percolation network of pores

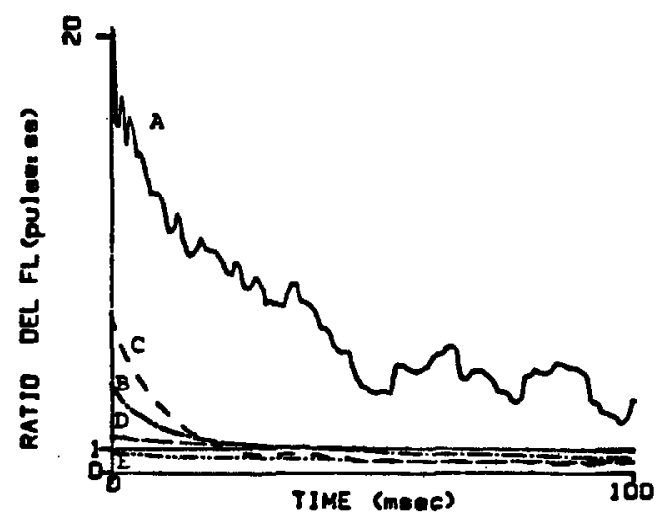

Fig. 8. Ratio of delayed fluorescences: pulsed versus steady-state excitation, with equalized initial phosporescence intensities. (A) Naphthalene in porous Vycor glass. (B) Naphthalene powder. (C) $5 \%$ isotopic mixed naphthalene crystal. (D) $11 \%$ isotopic mixed crystal. (E) $39 \%$ isotopic mixed crystal. is not consistent with our naphthalene in Vycor results.

In summary, our experiments demonstrate a nonrandom distribution for the triplet exciton populations in naphthalene samples that are not pure and perfect bulk crystals: crystalline powders, naphthalene filaments in porous silica and isotopic alloys with compositions below percolation. However, for isotopic alloys with compositions well above percolation the exciton distribution is random, within our experimental accuracy. The naphthalene "worms" inside the Vycor pores exhibit a one-dimensional topology, with filament lengths of about 200-1000. . The simulation results are consistent with the experimental ones. Furthermore, for one-dimensional topologies they show Wigner-like steady-state reactant distributions, implying the existence of steady-state, grating-like exciton quasi-superlattices in the excited naphthalene filaments.

\section{Summary and conclusions}

Both short-time and long-time techniques are in excellent agreement for the naphthalene impregnated Vycor samples. They both point towards the one-dimensional nature of the pores and filaments, over 10-100 nm length scales. We note that the Vycor pores are about three times narrower than the thinnest polycarbonate pores, and thus more suited for the "short-time" technique. However, preliminary short-time experiments on polycarbonate impregnated naphthalene still exhibit a one-dimensional behavior for the thinnest wires but not for the thicker ones. In the future, we plan to relate, quantitatively, experimental time scales and length scales. Overall, we have demonstrated the applicability and versatility of the exciton annihilation techniques. We have also demonstrated the utility of porous media as templates for one-dimensional solids. In addition, we have verified quantitatively the fractal-like (low-dimensional) reaction kinetics formalisms. Finally, we have shown that the compact diffusion in low dimensions leads to ordered rather than random steady-state distributions of reactant populations, giving, for the one-dimensional lattice, an effective excitation grating with a mean spacing as small as $50 \AA$. 


\section{Acknowledgement}

This work was supported by NSF Grant No. DMR8303919.

\section{References}

[1] K. Lakatos-Lindenberg and K.E. Shuler, J. Math. Phys. 12 (1971) 633.

[2] S. Alexander, J. Bernasconi, W.R. Schneider and R. Orbach, Rev. Mod. Phys. 53 (1981) 175.

[3] R. Zwanzig, J. Stat. Phys. 28 (1982) 127.

[4] V.M. Agranovich and M.D. Galanin, Electronic excitation energy transfer in condensed matter (North-Holland, Amsterdam, 1982).

[5] A. Blumen, J. Klafter and G. Zumofen, in: Optical spectroscopy of glasses, ed. I. Zschokke (Reidel, Dordrecht, 1986) p. 199.

[6] M. Buttiker, Y. Imry, R. Landauer and S. Pinhas, Phys. Rev. B 31 ( 1985) 6207.

[7] A. Benoit, C.P. Umbach, R.B. Laibowitz and R.A. Webb, Phys. Rev. Letters 58 (1987) 2343.

[8] D. Lenstra and W. van Haeringen, Phys. Rev. Letters 57 (1986) 1623.

[9] D.J. Thouless, Phys. Rev. Letters 39 (1977) 1167.

[10] J. Hoshen and R. Kopelman, J. Chem. Phys. 61 (1974) 330.

[11] R. Kopelman, in: Modern problems in solid state physics, Vol. 4, eds. V.M. Agranovich and R.M. Hochstrasser (NorthHolland, Amsterdam, 1983) p. 139.

[12] D.W. Schaefer, B.C. Bunker and J.P. Wilcoxon, Phys. Rev. Letters 58 (1987) 284.

[13] U. Even, K. Rademann, J. Jortner, N. Manor and R. Reisfeld, Phys. Rev. Letters 58 (1987) 285; 42 (1984) 2164.

[14] W.D. Dozier, J.M. Drake and J. Klafter, Phys. Rev. Letters 56 (1986) 197.

[15] R. Kopelman, S. Parus and J. Prasad, Phys. Rev. Letters 56 (1986) 1742.

[16] C.L. Yang, P. Evesque and M.A. El-Sayed, J. Phys. Chem. 89 (1985) 3442.

[ 17 ] R.M. Hochstrasser and J.D. Whiteman, J. Chem. Phys. 56 (1972) 5945.

[18] M.D. Fayer, in: Modern problems in solid state physics, Vol. 4, eds. V.M. Agranovich and R.M. Hochstrasser (NorthHolland, Amsterdam, 1983) p. 139.

[19] M.J. Burns, W.K. Liu and A.H. Zewail, in: Modern problems in solid state physics, Vol. 4, eds. V.M. Agranovich and R.M. Hochstrasser (North-Holland, Amsterdam, 1983) p. 301.

[20] S.D.D.V. Rughooputh, D. Bloor, D. Phillips and B. Movaghar, Phys. Rev. B 35 (1987) 8103;

W.J. Rodriguez, R.A. Auerbach and G.L. McPherson, J. Chem. Phys. 85 (1986) 6442.
[21] L.W. Anacker and R. Kopelman, Phys. Rev. Letters 58 (1987) 289; J. Phys. Chem. 91 (1987) 5555;

K. Lindenberg, B.J. Westand and R. Kopelman, Phys. Rev. Letters 60 (1988) 1777.

[22] Z. Racz, Phys. Rev. Letters 55 (1985) 1707.

[23] L.W. Anacker and R. Kopelman, J. Chem. Phys. 81 (1984) 6402.

[24] J.S. Newhouse and R. Kopelman, Phys. Rev. B 31 (1985) 1677.

[25] J.S. Newhouse and R. Kopelman, J. Chem. Phys. 85 (1986) 6804.

[26] R. Kopelman, J. Stat. Phys. 42 (1986) 185.

[27] R. Kopelman and S.J. Parus, in: Fractal aspects of materials. II, eds. D.W. Schaefer, R.B. Laibowitz, B.B. Mandelbrot and S.H. Liu (Materials Research Society, Boston, 1986) p. 50.

[28 ] R. Kopelman, in: Fractal aspects of materials. III, eds. A.J. Hurd, D.A. Weitz and B.B. Mandelbrot (Materials Research Society, Boston, 1987) p. 112.

[29] D.C. Torney and H.M. McConnel, J. Phys. Chem. 87 (1983) 1441.

[30] P.V. Elyutin, J. Phys. C 17 (1987) 1867.

[31] P. Evesque and J. Duran, J. Chem. Phys. 80 (1984) 3016.

[32] G. Zumofen, A. Blumen and J. Klafter, J. Chem. Phys. 82 (1985) 3198.

[33] R. Kopelman, J. Hoshen, J.S. Newhouse and P. Argyrakis, J. Stat. Phys. 30 (1983) 355.

[34] P.W. Klymko and R. Kopelman, J. Luminescence 24/25 (1981) 457.

[35] P.W. Klymko and R. Kopelman, J. Phys. Chem. 86 (1982) 3686.

[36] P.W. Klymko and R. Kopelman, J. Phys. Chem. 87 ( 1983 ) 4565.

[37] R. Kopelman, Science 241 (1988); P. Argyrakis and R. Kopelman, J. Phys. Chem. 91 (1987) 2699.

[38 ] S. Alexander and R. Orbach, J. Phys. (Paris) Lett. 43 (1982) L625.

[39] R. Rammal and G. Toulouse, J. Phys. (Paris) Lett. 44 (1983) L13.

[40] J. Prasad and R. Kopelman, J. Phys. Chem. 91 (1987) 265.

[41] W.D. Williams and N. Giordano, Rev. Sci. Instr. 55 (1984) 410.

[42] R. Kopelman, L. Li, S.J. Parus and J. Prasad, J. Luminescence 38 (1987) 289.

[43] A. Boukenter, B. Champagnon, E. Duval, J. Dumas, J.F. Quinson and J. Serughetti, Phys. Rev. Letters 57 (1986) 2391.

[44] P. Hertz, Math. Ann. 67 (1909) 387.

[45] D. Delande and J.C. Gay, Phys. Rev. Letters 57 (1986) 2006, 2877.

[46] M.L. Mehta, Random matrices and the statistical theory of energy levels (Academic Press, New York, 1967).

[47] J. Prasad and R. Kopelman. Phys. Rev. Letters 59 (1987) 2103. 